\title{
A new prescription for viscosity in Smoothed Particle Hydrodynamics
}

\author{
S.J. Watkins ${ }^{\star}$, A.S. Bhattal, N. Francis, J.A. Turner and A.P. Whitworth \\ Department of Physics and Astronomy, University of Wales, Cardiff, CF2 3BY, Wales, UK
}

Received October 2, 1995; accepted February 21, 1996

\begin{abstract}
Smoothed particle hydrodynamics (SPH) is a particle method for modelling hydrodynamical flows that has been successfully applied to a wide range of astrophysical problems. One of its main weaknesses, however, has been its inability to treat viscosity in a rigorous manner. We present a new method that can be used to solve the Navier-Stokes equation for an arbitrary viscosity. We compare the accuracy of the method to alternative methods for treating viscosity in SPH, and apply the method to a series of tests for which there exist analytic solutions. We find that the new method is significantly more accurate than other existing methods, computationally efficient, and that the results of simulations carried out using the method are in excellent agreement with the theory.
\end{abstract}

Key words: hydrodynamics — methods: numerical

\section{Introduction}

Smoothed particle hydrodynamics (SPH) is a fully Lagrangian particle method that was introduced by Lucy (1977) and Gingold \& Monaghan (1977) in order to simulate astrophysical phenomena which involve large densitycontrasts and complicated non-symmetric geometry. SPH is a very powerful technique that has been successfully applied to a wide range of problems. Until now, however, it has not been possible to treat viscous interactions properly in SPH. Instead, an artificial viscosity is used, the most common form being that due to Monaghan (1989), which is designed to prevent interpenetration of colliding streams and to resolve shock fronts; however, this artificial viscosity does not allow the specification of shear and bulk viscosity as separate parameters. Thus, for example, in the simulations of star formation of Turner et al. (1995) and Whitworth et al. (1995a) protostellar discs are formed, but their internal evolution cannot be followed due to the high effective shear viscosity.

Viscosity plays an important rôle in many astrophysical phenomena, from accretion discs to outflows. A method for correctly modelling viscosity in SPH therefore has the potential to increase greatly the range of problems to which SPH can be applied.

Some authors (e.g. Artymowicz \& Lubow 1994) have derived an empirical viscosity from the artificial viscosity, and recently attempts have been made to apply the

Send offprint requests to: S. Watkins

*e-mail: S.Watkins@astro.cf.ac.uk
SPH formalism to the Navier-Stokes equation (Flebbe et al. 1994; Takeda et al. 1994). The method of Takeda et al. can only be used in the case where the coefficient of shear viscosity, $\mu_{\mathrm{s}}$, is constant, and does not allow for the treatment of bulk viscosity. Thus the method of Flebbe et al. is the only existing method that allows the modelling of a general viscosity in SPH.

The method we present here is also based on the Navier-Stokes equation. In Sect. 2 we derive the method. In Sect. 3 we apply the new method to a series of simple static tests, and compare its accuracy with that of other methods. In Sect. 4 we apply the method to a series of dynamic simulations for which there exist analytic solutions, testing its ability to model both shear and bulk viscosity. Finally, in Sect. 5 we present our conclusions.

\section{Method}

The acceleration of an element in a fluid is given by the Navier-Stokes equation which can be written using the standard index-summation convention as:

$$
\begin{aligned}
\rho \frac{\mathrm{D} v_{\alpha}}{\mathrm{D} t}= & \rho F_{\alpha}-\frac{\partial p}{\partial x_{\alpha}}+\frac{\partial}{\partial x_{\beta}}\left\{\mu_{\mathrm{s}} \frac{\partial v_{\alpha}}{\partial x_{\beta}}+\mu_{\mathrm{s}} \frac{\partial v_{\beta}}{\partial x_{\alpha}}\right\} \\
& +\frac{\partial}{\partial x_{\alpha}}\left\{\left(\mu_{\mathrm{b}}-\frac{2}{3} \mu_{\mathrm{s}}\right) \frac{\partial v_{\gamma}}{\partial x_{\gamma}}\right\},
\end{aligned}
$$

where $\frac{\mathrm{Dv}}{\mathrm{D} t}$ is the rate of change of velocity following the fluid flow, $\mu_{\mathrm{b}}$ is the coefficient of bulk viscosity and $\mu_{\mathrm{s}}$ is 
the coefficient of shear viscosity. In vector form the equation is:

$$
\begin{aligned}
\rho \frac{\mathrm{D} \mathbf{v}}{\mathrm{D} t}= & \rho \mathbf{F}-\nabla p-\mu_{\mathrm{s}} \nabla \times \nabla \times \mathbf{v}+\left(\mu_{\mathrm{b}}+2 \mu_{\mathrm{s}}\right) \nabla(\nabla \cdot \mathbf{v}) \\
& +(\nabla \cdot \mathbf{v}) \nabla \mu_{\mathrm{b}}+2\left(\nabla \mu_{\mathrm{s}} \cdot \nabla\right) \mathbf{v} \\
& +\nabla \mu_{\mathrm{s}} \times(\nabla \times \mathbf{v})-\frac{2}{3} \nabla\left(\mu_{\mathrm{s}} \nabla \cdot \mathbf{v}\right)
\end{aligned}
$$

Thus in order to evaluate the viscous forces on an SPH particle, it is necessary to be able to calculate accurately the second derivatives of the velocity field.

The method presented here is very simple to implement as it is based directly upon the standard SPH expressions for first derivatives (see e.g. Monaghan 1992). For instance, a standard expression for $\nabla \times \mathbf{v}$ is:

$$
\rho_{i}(\nabla \times \mathbf{v})_{i}=\sum_{j} m_{j}\left(\mathbf{v}_{i}-\mathbf{v}_{j}\right) \times \nabla_{i} W_{i j}
$$

We find that more accurate results are obtained if the derivative is re-written using $\rho_{j}$ instead of $\rho_{i}$ :

$$
(\nabla \times \mathbf{v})_{i}=\sum_{j} \frac{m_{j}}{\rho_{j}}\left(\mathbf{v}_{i}-\mathbf{v}_{j}\right) \times \nabla_{i} W_{i j} .
$$

This method can be used to calculate $\nabla \times \mathbf{v}$ at each particle, and the process then repeated to calculate $\nabla \times \nabla \times \mathbf{v}$ :

$$
(\nabla \times \nabla \times \mathbf{v})_{i}=\sum_{j} \frac{m_{j}}{\rho_{j}}\left[(\nabla \times \mathbf{v})_{i}-(\nabla \times \mathbf{v})_{j}\right] \times \nabla_{i} W_{i j}
$$

A similar process can be applied to calculate the other terms in the Navier-Stokes equation; for instance, $\nabla \cdot \mathbf{v}$ can be calculated using:

$$
(\nabla \cdot \mathbf{v})_{i}=\sum_{j} \frac{m_{j}}{\rho_{j}}\left(\mathbf{v}_{j}-\mathbf{v}_{i}\right) \cdot \nabla_{i} W_{i j} .
$$

The method for calculating $\left(\nabla \mu_{\mathrm{s}} \cdot \nabla\right) \mathbf{v}$ is first to calculate $\nabla \mu_{\mathrm{s}}$ :

$$
\left(\nabla \mu_{\mathrm{s}}\right)_{i}=\sum_{j} \frac{m_{j}}{\rho_{j}}\left[\left(\mu_{\mathrm{s}}\right)_{j}-\left(\mu_{\mathrm{s}}\right)_{i}\right] \nabla_{i} W_{i j}
$$

This is then substituted into the following expression:

$$
\left[\left(\nabla \mu_{\mathrm{s}} \cdot \nabla\right) \mathbf{v}\right]_{i}=\sum_{j} \frac{m_{j}}{\rho_{j}}\left(\mathbf{v}_{j}-\mathbf{v}_{i}\right)\left[\left(\nabla \mu_{\mathrm{s}}\right)_{i} \cdot \nabla_{i} W_{i j}\right]
$$

Thus, the viscous acceleration on an SPH particle can be calculated using simple, standard methods. The simplicity of this method is also its strength, as it can easily be applied to calculate any second derivatives.

\subsection{Bulk viscosity}

The most common form of the artificial viscosity (Monaghan 1989) is

$$
\mathbf{a}_{\mathrm{visc}}=-\sum_{j} m_{j} \Pi_{i j} \nabla_{i} W_{i j},
$$

where

$$
\Pi_{i j}= \begin{cases}\frac{-\alpha \bar{c}_{i j} \mu_{i j}+\beta \mu_{i j}^{2}}{\bar{\rho}_{i j}} & \mathbf{v}_{i j} \cdot \mathbf{r}_{i j}<0, \\ 0 & \mathbf{v}_{i j} \cdot \mathbf{r}_{i j}>0,\end{cases}
$$

and

$$
\mu_{i j}=\frac{h \mathbf{v}_{i j} \cdot \mathbf{r}_{i j}}{\mathbf{r}_{i j}+0.01 h^{2}} .
$$

Here $\bar{c}_{i j}$ is the mean value of the sound speed at particles $i$ and $j$. The $\alpha$-term is equivalent to a combined NavierStokes shear and bulk viscosity (Meglicki et al. 1993), and so can be replaced by a proper Navier-Stokes viscosity calculated using the new method, while the $\beta$-term is similar to a Von Neumann-Richtmyer viscosity, and is needed to prevent penetration in high Mach shocks. When modelling such shocks with the new method, therefore, it is necessary to retain the $\beta$-term from the artificial viscosity. This does not compromise the viscous forces calculated using the new method, as the $\beta$-term is only significant for particles that are approaching each other very fast and almost directly head-on.

\section{Static tests}

In order to test the accuracy of the new method for calculating the viscous terms, it was tested on a series of simple velocity fields. The results were compared with those obtained using two other methods, due to Flebbe et al. (1994) and Brookshaw (1985). The Flebbe method allows one to calculate all of the terms in the Navier-Stokes equation, whereas the Brookshaw method only gives $\nabla^{2} \mathbf{v}$, and so can only be used for modelling viscosity in certain artificial situations, but it is shown here for comparison.

The methods were first tested on a uniform density distribution of particles given a simple velocity field, for which the results calculated using the new method could be easily compared with known analytic values. Tests were carried out in both two and three dimensions.

5000 particles were distributed with random positions in a box of unit side, and then settled to a uniform density distribution. The box was given periodic boundary conditions in order to avoid errors being introduced at the edges. The particles were given a uniform, constant $h$ based on the density set to give them on average 30 neighbours within their kernel for the $2 \mathrm{D}$ case and 60 neighbours for the 3D case (see Appendix A for details of the $h$-setting). The densities at the particle positions for the 3D case are shown in Fig. 1a; the mean density is $\bar{\rho}=0.999$ and the standard deviation is $\sigma_{\rho}=0.004$. 

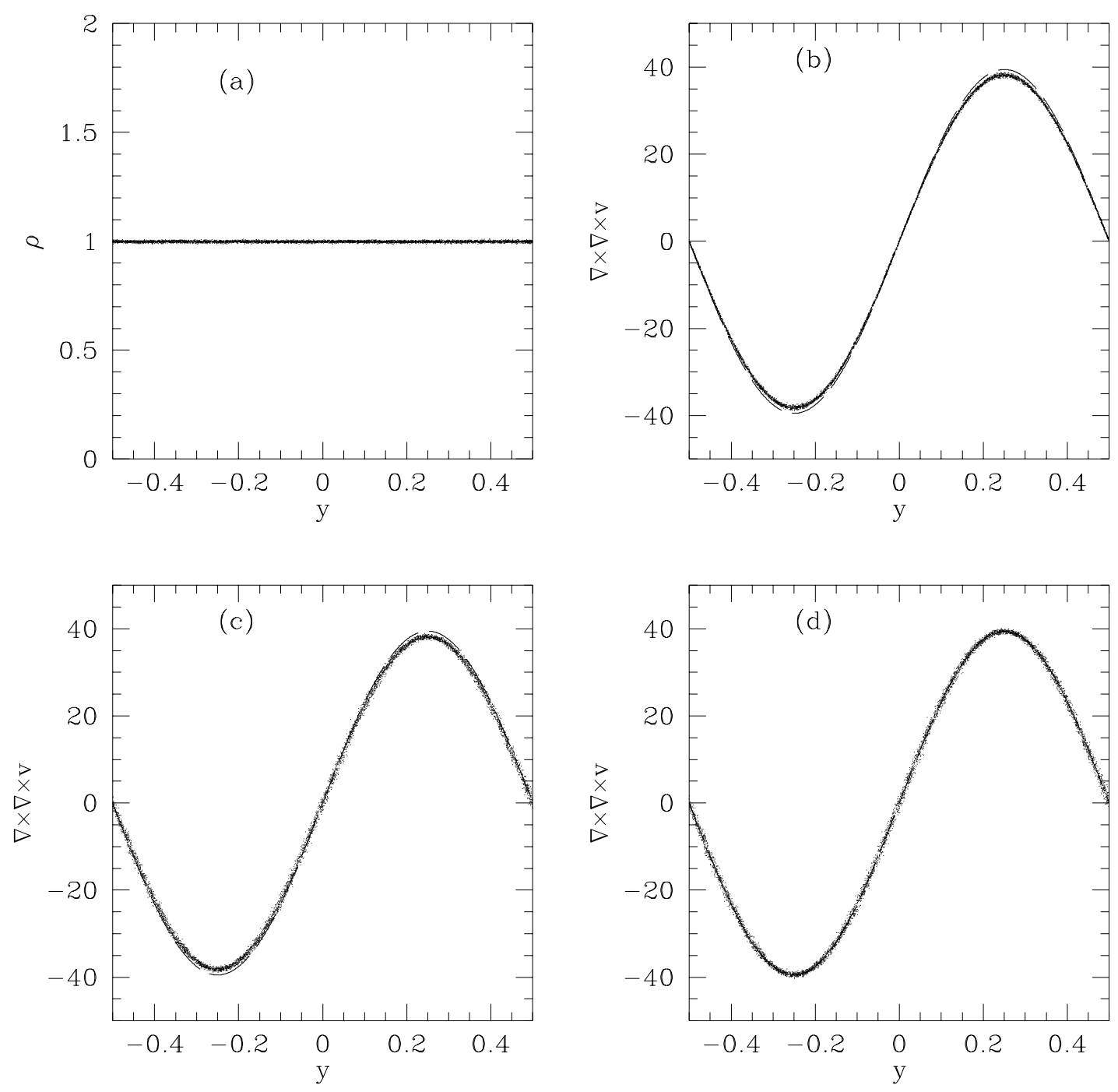

Fig. 1. Results for 5000 particles settled to uniform density in a 3D box. All plots are at particle positions. a) shows the density as a function of $y$. b)-d) show the $x$-component of $\nabla \times \nabla \times \mathbf{v}$ calculated using $\mathbf{b}$ ) the new method, $\mathbf{c}$ ) the Flebbe method, and d) the Brookshaw method

The periodic boundary conditions mean that any velocity field given to the particles must also be periodic, as otherwise there will be a discontinuity in the velocity at the boundary and the derivatives of the velocity will not be well-behaved. The following velocity field was used:

$$
v_{x}=\sin (2 \pi y),
$$

with all other components of the velocity set to zero. The second derivatives of the velocity field then have the following form:

$$
\begin{aligned}
\nabla(\nabla \cdot \mathbf{v}) & =\mathbf{0}, \\
\nabla \times \nabla \times \mathbf{v} & =\left(4 \pi^{2} \sin (2 \pi y), 0,0\right) .
\end{aligned}
$$

In this case $\nabla \times \nabla \times \mathbf{v}$ can be calculated using the new method, the Flebbe method, and also, since $\nabla(\nabla \cdot \mathbf{v})=\mathbf{0}$, the Brookshaw method. The results obtained in the 3D case are shown in Figs. 1b-d respectively. All of the methods are very accurate, with the new method and Flebbe method, both of which require two sums over neighbours, being slightly over-smoothed at the peaks of the derivatives.

In a real simulation, the velocity field is unlikely to be free from noise. The methods were therefore tested on the same settled distribution of particles, with a random component added to the velocity of each particle. The $x$-component of the velocity at the particles is shown in Fig. 2a. The values of $\nabla \times \nabla \times \mathbf{v}$ calculated using the new, Flebbe and Brookshaw methods are shown in Figs. 2b-d respectively. The new method is the most accurate, with the Flebbe method also being very accurate, whilst the Brookshaw method is extremely inaccurate. 

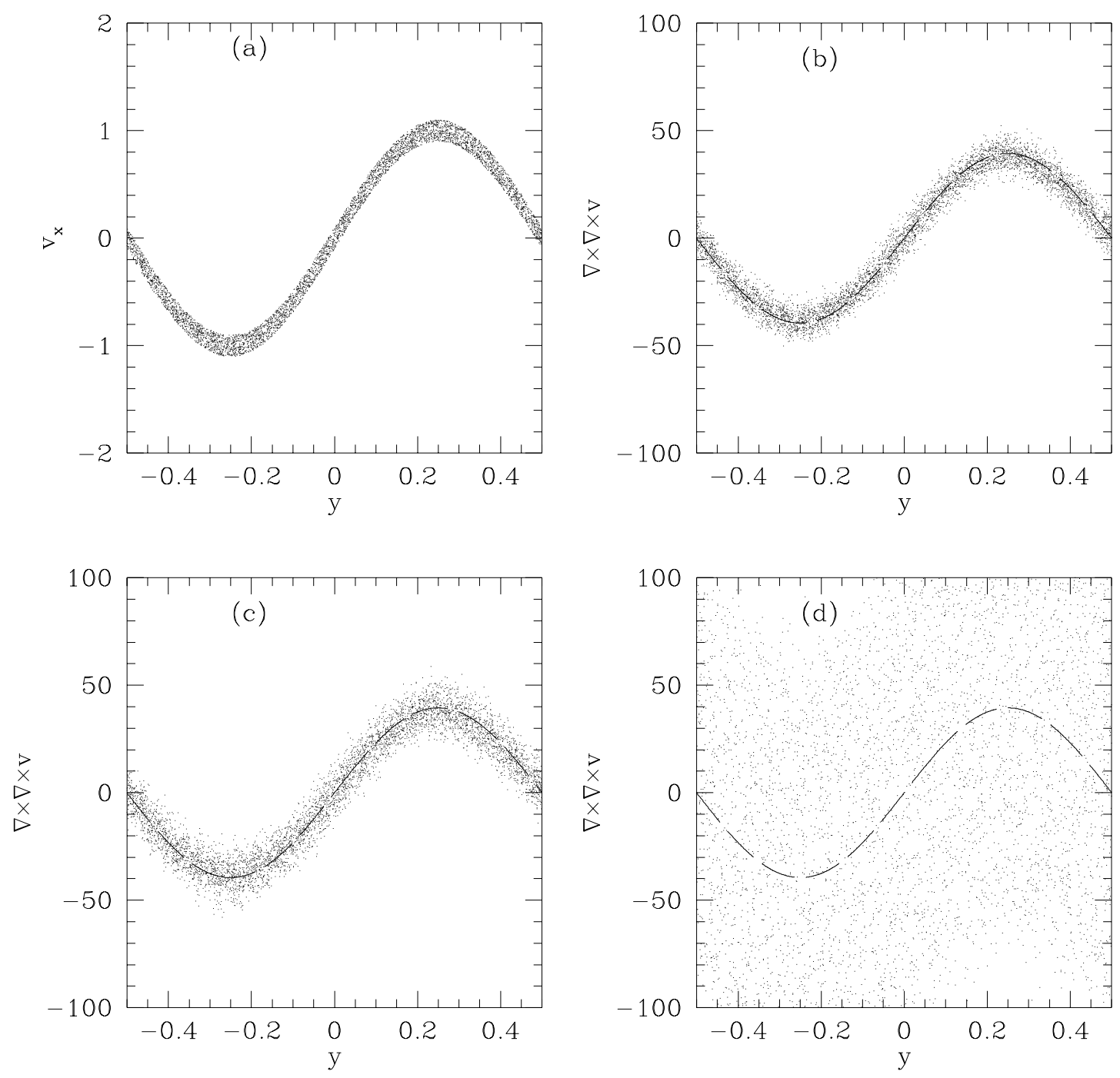

Fig. 2. Results for 5000 particles settled to uniform density in a 3D box, given a noisy velocity field. All plots are at particle positions. a) shows the $x$-velocity as a function of $y$. b)-d) show the $x$-component of $\nabla \times \nabla \times \mathbf{v}$ calculated using $\mathbf{b}$ ) the new method, c) the Flebbe method, and d) the Brookshaw method

It is a more rigorous test of the methods to see how accurately they calculate the derivatives in a case where the particles are not settled. The methods were therefore tested on 5000 particles distributed randomly in a 3D box but not settled, with constant $h$ as before to give about 60 neighbours. The densities at the particle positions are shown in Fig. 3a. As this is a random distribution, the self-density of each particle is not included in its density calculation (see Whitworth et al. 1995b; Wood 1981). The mean density is $\bar{\rho}=1.013$ and the standard deviation is $\sigma_{\rho}=0.281$. The values of $\nabla \times \nabla \times \mathbf{v}$ obtained with the new method, the Flebbe method and the Brookshaw method are shown in Figs. 3b-d respectively. The new method is the most accurate method by far, with the other methods giving very poor results. We note that the noise in the second derivative calculated using the new method is considerably less than the noise in the density.

The results presented in this section lead us to conclude that the new method is very accurate in a simple case, and considerably more accurate than the method of Flebbe et al. in more realistic cases with noise. In Sect. 4 we proceed to test the new method on a series of dynamic problems.

\section{Dynamic tests}

\subsection{Poiseuille flow}

Two-dimensional Poiseuille flow is the flow of a fluid between two parallel plates with a uniform and constant acceleration $g$ in the direction of the flow. The coefficient of shear viscosity, $\mu_{\mathrm{s}}$, is constant, and there is no bulk 

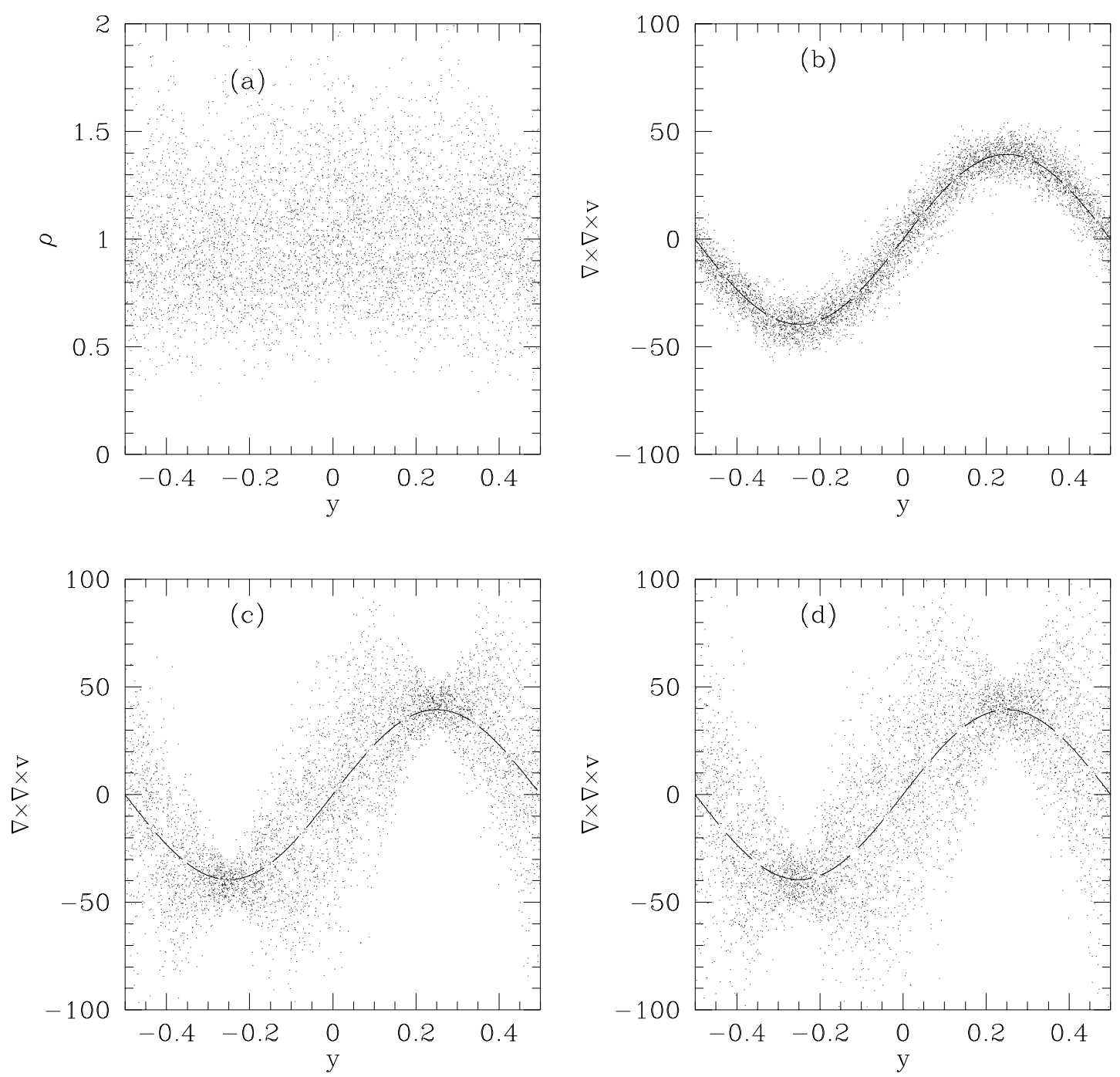

Fig. 3. Results for 5000 particles distributed randomly in a 3D box. All plots are at particle positions. a) shows the density as a function of $y$. b)-d) show the $x$-component of $\nabla \times \nabla \times \mathbf{v}$ calculated using $\mathbf{b}$ ) the new method, $\mathbf{c}$ ) the Flebbe method, and $\mathbf{d}$ ) the Brookshaw method

viscosity. The velocity profile of the fluid should evolve towards a stationary state under a constant acceleration and shear viscosity. By the velocity profile we mean $v_{x}$ as a function of $y$.

If the flow is in the $x$-direction and the plates are in the planes $y= \pm a$, then the stationary solution is:

$$
v_{x}=\frac{g\left(a^{2}-y^{2}\right)}{2 \mu_{\mathrm{s}}} .
$$

The simulation was carried out using 4900 particles distributed in 2D on a grid of unit side, centred on the origin. Periodic boundary conditions were applied in the $x$-direction. Particles in the range $-0.4 \leq y \leq 0.4$ were set to be active particles, while particles outside this range were set to be boundary particles. The boundary particles are given the analytic density, $\rho=1$, and a pseudo-velocity (see below), but are not moved during the course of the simulation.

\subsubsection{Boundary conditions}

In order for the velocity derivatives of particles near the boundary to be calculated accurately, it is necessary for the boundary particles to be given a pseudo-velocity. The sole purpose of this velocity is to enable the correct calculation of the derivatives for the boundary particles, and the boundary particles are not actually moved. If the boundary particles are not given this velocity then the velocity field for particles near the boundary is poorly sampled and hence errors are introduced into the derivatives. For the same reason, when calculating $\nabla \times \nabla \times \mathbf{v}$, it is necessary to give the boundary particles a value of $\nabla \times \mathbf{v}$. 


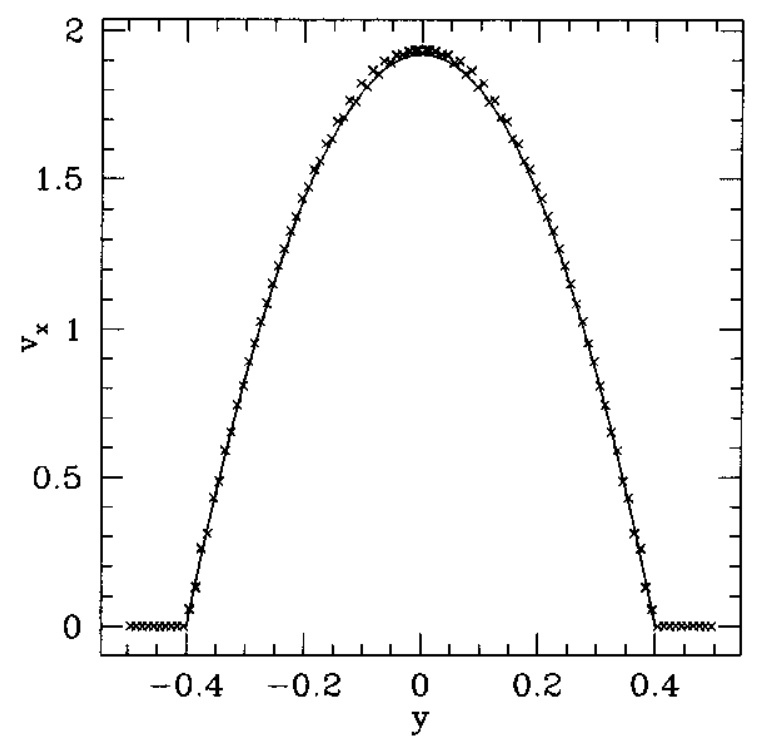

Fig. 4. Poiseuille flow: the steady-state $y$-velocity plotted at particle $x$-positions. The analytic profile is shown as a solid line

If particle $i$ has a neighbour $j$ that is a boundary particle, then the velocity at $j$ is extrapolated from the velocity at $i$ by assuming that the velocity profile is parabolic near the boundary:

$$
\begin{aligned}
& v_{x}=K\left(a^{2}-y^{2}\right), \\
& v_{y}=0
\end{aligned}
$$

so that:

$$
\left[v_{x}\right]_{j}=\frac{\left[v_{x}\right]_{i}\left(a^{2}-y_{j}^{2}\right)}{a^{2}-y_{i}^{2}}
$$

This satisfies the no-slip condition $\left(v_{x}=0\right.$ at $\left.y= \pm a\right)$.

If the velocity profile near the boundary is parabolic, then $\nabla \times \mathbf{v}$ is in the $z$-direction and is a linear function of the $y$-position:

$$
\nabla \times \mathbf{v}=(0,0,-2 K y),
$$

so that $\nabla \times \mathbf{v}$ at $j$ can be extrapolated from the value at $i$ :

$$
(\nabla \times \mathbf{v})_{j}=\frac{y_{j}(\nabla \times \mathbf{v})_{i}}{y_{i}}
$$

The following values were used in the simulation: $a=$ $0.4, g=3.6, \mu_{\mathrm{s}}=0.15$, giving a Reynolds number $R e \sim 5$. The particles were given a uniform and constant value of $h$ chosen to give each particle 30 neighbours.

\subsubsection{Results}

The simulation is started with the fluid initially at rest. The particles are free to move anywhere in the computational domain. The particles begin to move under the influence of the constant acceleration $g$ and viscous forces, and eventually the velocity profile reaches a steady state. Figure 4 shows the stationary velocity profile obtained. After the velocity profile has reached and held the stationary state for a time, an instability develops in which neighbouring rows of particles develop velocities that are alternately higher and lower than the corresponding stationary velocity. This instability rapidly grows and corrupts the solution. If the particles are restricted to moving only in the $x$-direction then the growth of this instability is suppressed, and the velocity profile maintains the stationary state for more than a thousand timesteps.

Poiseuille flow becomes turbulent for a Reynolds number $R e>10-100$. As neither SPH nor any other numerical method can accurately follow turbulent flow, it should be expected that a simulation of such a flow should become unstable. We have carried out a simulation of a flow with a Reynolds number $R e \sim 20$, and find that the flow evolves towards the stationary solution, but that as it approaches the solution an instability develops at the boundary which rapidly spreads throughout the computational domain, corrupting the results.

\subsection{Viscous evolution of a ring of gas}

The self-similar solution for the secular evolution of a ring of matter around a central gravitating object derived by Lynden-Bell \& Pringle (1974) is a good test of how accurately the new method models shear viscosity in dynamic situations.

If a ring of mass $m$ is at an initial radius $R_{0}$ then in terms of dimensionless radius $x=R / R_{0}$ and time $\tau=$ $12 \nu t R_{0}^{-2}$ with $\nu$ being the kinematic viscosity, the surface density is given by:

$$
\sigma(x, \tau)=\left(\frac{m}{\pi R_{0}^{2}}\right) \tau^{-1} x^{-\frac{1}{4}} \exp \left[-\frac{\left(1+x^{2}\right)}{\tau}\right] I_{\frac{1}{4}}\left(\frac{2 x}{\tau}\right)
$$

where $I_{\frac{1}{4}}$ is a modified Bessel function (Pringle 1981).

The modelling of this solution is a good test of the accuracy of the new method. In particular, the ring should evolve on the correct viscous timescale.

\subsubsection{Initial Conditions}

The initial conditions were chosen to be similar to those of Maddison et al. (1996), with particles placed in concentric rings to give a uniform distribution of particles, and then each particle given a mass proportional to the surface density at that point, $\sigma(r)$. The disadvantage of doing this is that the most massive particles can have a very large self-density. This means that the evolution of the ring cannot be followed beyond the stage where the density of the particles approaches this minimum density. The evolution of the system can be followed further by using more particles, so that the mass per particle is smaller, and/or a larger smoothing length, $h$, so that the self-density of the 
particles is smaller. This is unfortunate as it would be preferable to show that the new method can follow the evolution of the system with as few particles and as small a value of $h$ as possible. We have chosen to use 14500 particles and $h=0.045$ in order to enable the solution to be modelled to the same time as that of Flebbe et al., who have carried out the same test; we have, however, also tested the method using fewer particles and a smaller smoothing length, and find that the results are as accurate as those presented here until the self-density halts the evolution of the system.

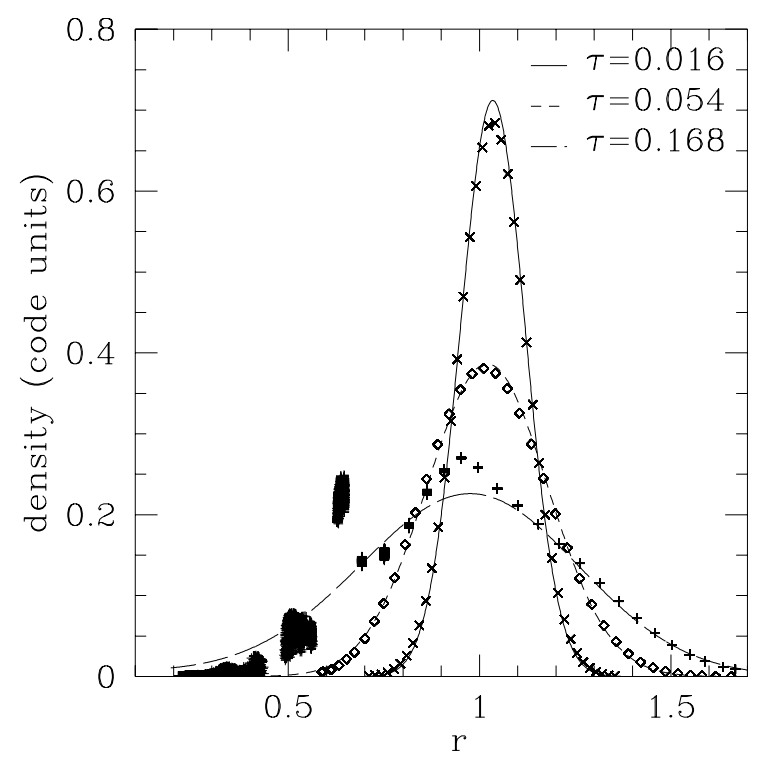

Fig. 5. Evolution of a ring of gas: density as a function of radius for $\nu=1.510^{-3}$. Densities are plotted at all particle positions. The analytic densities are shown as solid lines

The simulation was started at time $\tau=0.016$ in order to avoid the singularity at $\tau=0$. The initial velocity consists of the transverse velocity for a Keplerian orbit plus a radial term. The radial velocity in terms of $x$ and $\tau$ is:

$$
v_{\mathrm{r}}=-\frac{3 \nu}{R_{0}} \frac{\partial}{\partial x} \ln \left(x^{1 / 2} \sigma\right)
$$

(Frank et al. 1992). The forces acting on the gas particles are a gravitational attraction from the central point mass, and viscous forces that can be calculated from the NavierStokes equation.

\subsubsection{Equation of Motion}

The viscous forces on the particles can be calculated from the Navier-Stokes equation. In this case, the bulk viscosity $\mu_{\mathrm{b}}$ is zero, the kinematic shear viscosity, $\nu=\mu_{\mathrm{s}} / \sigma$ is constant, and $\nabla \cdot \mathbf{v}=0$ so that Eq. (2) can be rearranged to give:

$$
\frac{D \mathbf{v}}{D t}=\frac{\nu}{\sigma}\left\{\sigma \nabla^{2} \mathbf{v}+2(\nabla \sigma \cdot \nabla) \mathbf{v}+(\nabla \sigma) \times(\nabla \times \mathbf{v})\right\}
$$

$$
-\frac{G M \mathbf{r}}{r^{3}}
$$

where $M$ is the mass of the central object. The above terms can all be calculated using the new method.

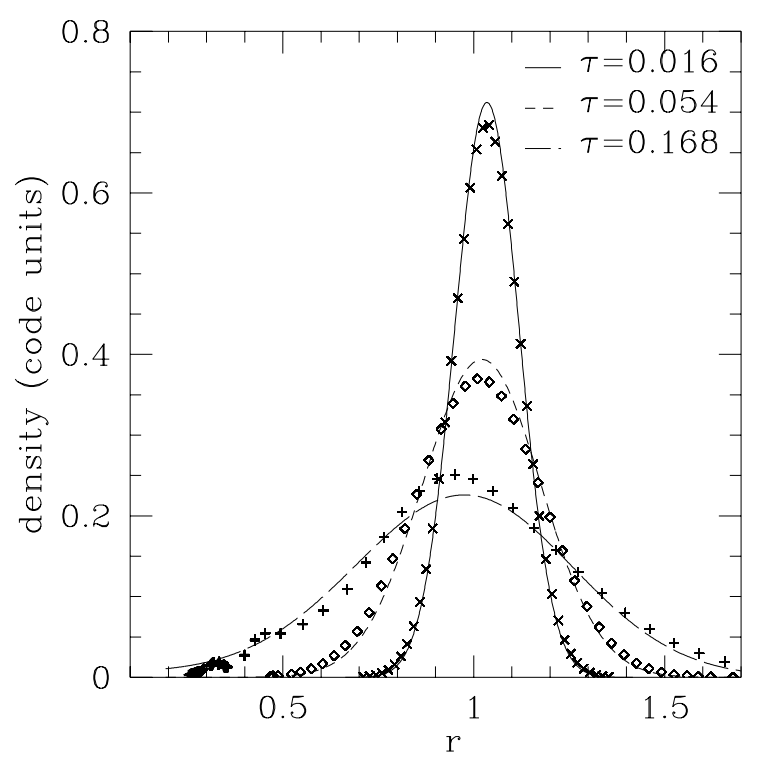

Fig. 6. Evolution of a ring of gas: density as a function of radius for $\nu=4.510^{-3}$. Densities are plotted at all particle positions. The analytic densities are shown as solid lines

\subsubsection{Results}

The following parameters were used in the simulation: $G=1, M=1, R_{0}=1$, and $\nu=1.510^{-3}$. The results are shown in Fig. 5. We find that after approximately ten orbital periods, an instability develops at the inner edge, in which the inner rings begin to oscillate in and out, driving density waves through the solution. We note that the results of Flebbe et al. appear to exhibit the same oscillations. The inner rings then begin to break up into clumps, in what may be the same instability as that identified by Maddison et al. (1996).

It is possible to overcome this instability by increasing the strength of the viscosity, so that the system evolves on a faster timescale. The Lynden-Bell \& Pringle solution, however, depends on the system evolving on a secular rather than orbital timescale, and so for higher viscosity the evolution of the system will not exactly match the analytic solution. The results for a simulation with $\nu=4.510^{-3}$ are shown in Fig. 6 . At early times the ring evolves slightly faster than the Lynden-Bell \& Pringle solution, but is able to evolve to the end of the simulation without being significantly corrupted.

\subsection{Riemann shock-tube}

The Riemann shock-tube is a dynamic non-gravitating hydrodynamic system. There is an analytic solution for the 

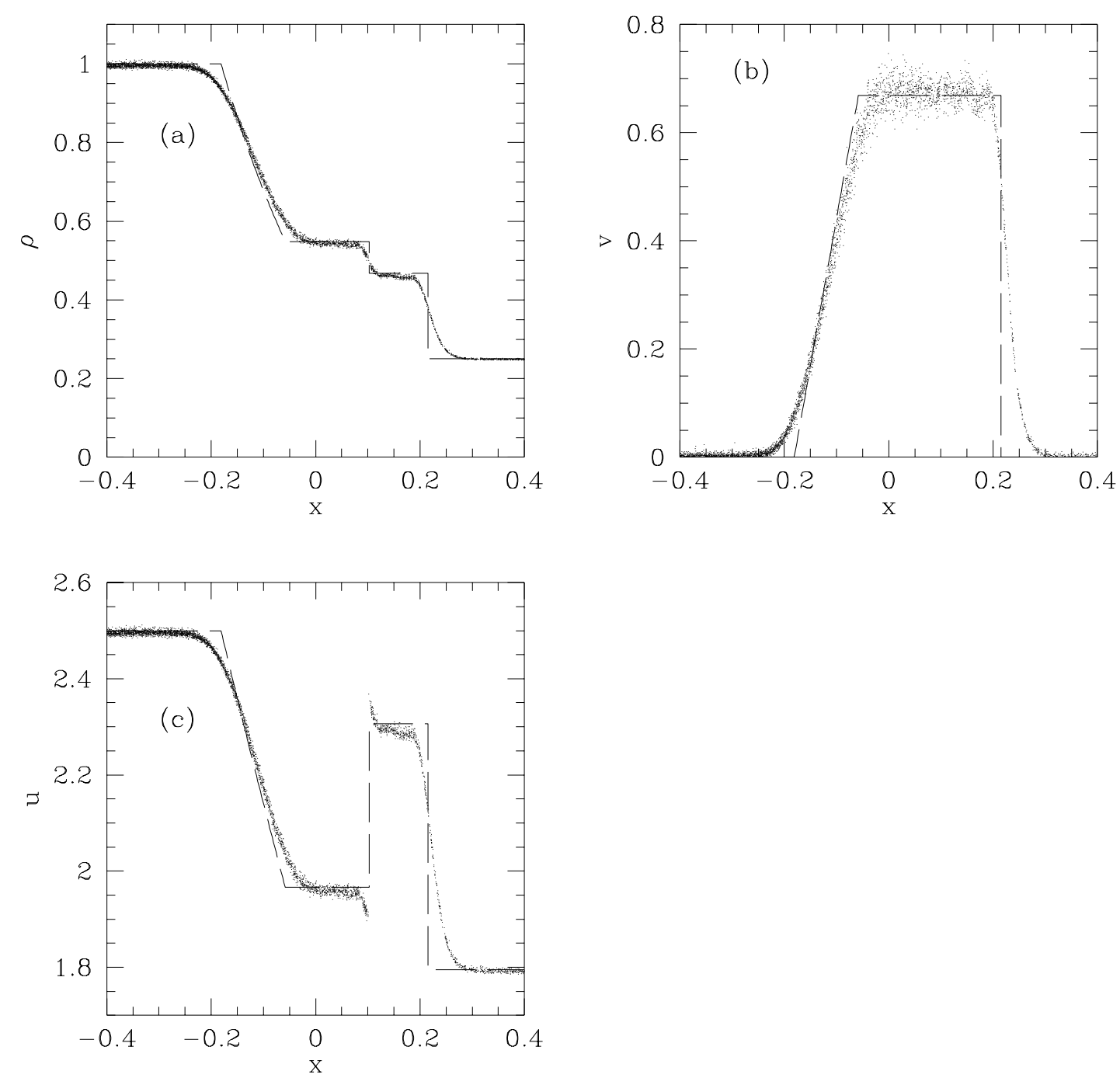

Fig. 7. Results for the Riemann shock-tube using the new method. a) is the density as a function of $x, \mathbf{b})$ is the $x$-velocity as a function of $x$, and $\mathbf{c}$ ) is the thermal energy as a function of $x$. The analytic values are shown as dashed lines

evolution of the system and it has been well-studied using both SPH (e.g. Monaghan \& Gingold 1983) and finite difference methods (Sod 1978). This system therefore provides not only a test of the ability of the new method to calculate bulk viscous forces accurately in a dynamic situation, but also an opportunity to compare the new method with other methods used in SPH, in particular the artificial viscosity introduced by Monaghan (1989).

The artificial viscosity was introduced into SPH in order to stop interpenetration of particles in colliding streams, and to resolve shocks. If the new method is to be used instead of the artificial viscosity, it must be capable of stopping penetration.

The shock-tube is a tube of gas which is separated by a membrane into two regions of different pressures and densities. Each region is in an equilibrium state of constant pressure, density and temperature. At the start of the simulation the membrane is removed. A shock moves into the low-density gas and a rarefaction wave travels into the higher density gas. A contact discontinuity forms near the centre and travels into the low-density region behind the shock.

\subsubsection{Initial conditions}

The initial conditions were those used by Monaghan \& Gingold (1983), as listed below. Although most previous workers have modelled this system in $1 \mathrm{D}$, for which it is easy to obtain good results, we have carried out our simulations in $3 \mathrm{D}$, as we feel that it is a more appropriate test for a method which will be applied to 3D problems. The simulations were carried out with 2000 particles on the low-density side of the membrane and 8000 particles on the high-density side. $h$ was set to give each particle 50 neighbours. Periodic boundary conditions were used, with 
a periodic length of 2.0 in the $x$-direction, and 0.125 in the $y$ and $z$ directions, i.e. a long, thin tube. The membrane was in the plane $x=0$. A polytropic equation of state, $P=K \rho^{\gamma}$ was used, with the ratio of specific heats $\gamma=1.4$. The values of $K, P$ and $\rho$ used and the corresponding sound speeds $a_{0}$ are listed in Table 1 .

There is no shear viscosity in this simulation, so Eq. (2) becomes:

$$
\rho \frac{D \mathbf{v}}{D t}=-\nabla p+\mu_{\mathrm{b}} \nabla(\nabla \cdot \mathbf{v})+(\nabla \cdot \mathbf{v}) \nabla \mu_{\mathrm{b}}
$$

In the continuum limit the $\alpha$-term of the artificial viscosity is equivalent to a kinematic bulk viscosity $\nu_{\mathrm{b}}=\alpha a_{0} h / 8$ where $h$ is the SPH smoothing length, as well as a similar shear viscosity (Murray 1996). The results presented here are calculated using the same form for the bulk viscosity without the associated shear viscosity, and $\alpha=1$ so that $\mu_{\mathrm{b}}=\nu_{\mathrm{b}} \rho=a_{0} h \rho / 8$ at each particle.

Table 1. Values used in Riemann shock-tube

\begin{tabular}{lllll}
\hline & $P$ & $K$ & $\rho$ & $a_{0}^{2}$ \\
\hline High-Density & 1 & 1 & 1 & 1.4 \\
Low-Density & 0.1795 & 1.25 & 0.25 & 1.0052 \\
\hline
\end{tabular}

\subsubsection{Results}

The results are shown in Fig. 7. Plot (a) shows the density profile. The three regions of the rarefaction wave, contact discontinuity and shock are clearly visible. Plot (b) shows the $x$-velocity profile, and (c) shows the thermal energy as a function of $x$. The analytic values are shown as dashed lines. The corresponding results for the artificial viscosity can be found in Turner et al. (1995).

The results obtained match the analytic profiles closely. The contact discontinuity and shock are both accurately modelled. Both are smoothed over a range of about $2 h$. The edges of the rarefaction wave are similarly smoothed. There are some post-shock oscillations, but overall the results are in very good agreement with the analytic values, and of greater accuracy than the results obtained using the same code with the artificial viscosity by Turner et al. (1995). The main difference is that the double sum required for the new method means that the shocks are broadened over a scale of $\sim 2 h$, as compared with $\sim h$ for the artificial viscosity. The scatter about the analytic velocity solution is about $11 \%$ for the new method, compared with about $15 \%$ in the results of Turner et al. The only effect of the $\beta$-term in the simulation using the new method is slightly to reduce the post-shock oscillations.

We therefore conclude that the new method enables the accurate treatment of bulk viscosity in dynamic situations.

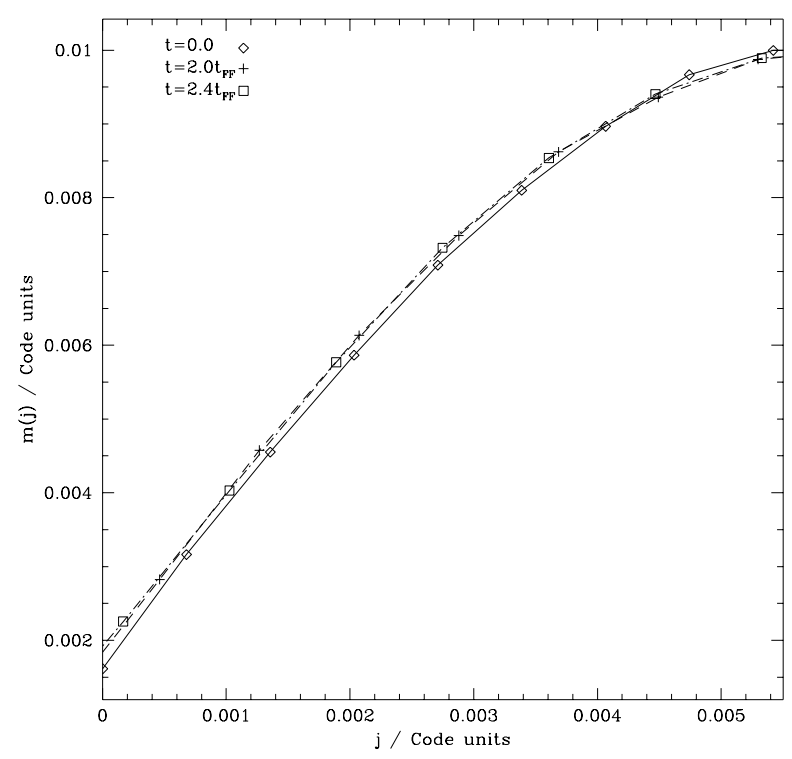

Fig. 8. Collapse of a rotating isothermal sphere: $m(j)$ vs. $j$ at $t=0, t=2.0 t_{\mathrm{FF}}, t=2.4 t_{\mathrm{FF}}$

\subsection{Collapse of a rotating, axisymmetric isothermal sphere}

The collapse of a rotating, axisymmetric isothermal sphere is a test proposed by Monaghan \& Lattanzio (1985). If $m(j)$ is the mass with specific angular momentum $\leq j$, then as there are no gravitational torques to redistribute angular momentum, the cumulative distribution of specific angular momentum should remain invariant throughout the collapse. This is a test of whether the viscosity causes spurious transport of angular momentum. The initial conditions are a $1 M_{\odot}$ cloud having uniform density $1.810^{-17} \mathrm{~g} \mathrm{~cm}^{-3}$ and of temperature $T=10 \mathrm{~K}$, ratio of thermal kinetic energy to gravitational energy $\alpha=0.25$, with a spherical reflecting boundary at $R \sim 2000 \mathrm{AU}$. The cloud is in solid-body rotation with the ratio of rotational energy to gravitational energy $\beta=0.20$.

The viscous forces were calculated using constant kinematic bulk viscosity, $\nu=310^{13} \mathrm{~m}^{2} \mathrm{~s}^{-1}$, chosen to be strong enough to stop the particles penetrating through each other once the sphere collapses down into a plane. The gravitational forces were calculated using a tree code (Barnes \& Hut 1986). The simulation used 2491 particles.

\subsubsection{Results}

Figure 8 shows $m(j)$ as a function of $j$ at the start of the simulation and after 2.0 and 2.4 freefall times, by which time the central density has increased to $1.310^{-14} \mathrm{~g} \mathrm{~cm}^{-3}$. It can be seen that there is very little change in the profile over the course of the simulation. We therefore conclude that the new method does not lead to unphysical transport of angular momentum. 


\section{Conclusions}

The new method has been used in both static and dynamic tests. In the static cases, it gives second derivatives to a high degree of accuracy and better than the other methods of which we are aware; in particular it is much more accurate for the case where there is noise in the density field.

In dynamic tests, the simulation of $2 \mathrm{D}$ Poiseuille flow evolves to the correct stationary solution, and the evolution of a ring of matter according to the Lynden-Bell/Pringle solution occurs on the correct viscous timescale, showing that the method gives accurate treatment of shear viscosity in dynamic situations. The results of the simulation of the Riemann shock-tube are of an accuracy similar to that obtained by other workers using the artificial viscosity of Monaghan (1989). The shock, contact discontinuity and rarefaction wave are all accurately modelled and there is very little interpenetration.

The new method has therefore been tested on both shear and bulk viscosity, and in both static and dynamic situations. In all cases, it has been shown to be at least as accurate as, and often more accurate than, any other method for treating viscosity in SPH. We therefore conclude that this method has the potential to be applied to a wide range of problems in SPH in which the correct treatment of both bulk and shear viscosity is important.

Acknowledgements. We would like to thank the anonymous referee for his helpful comments. SJW would like to thank Nick Moore for many enlightening discussions. SJW and ASB acknowledge the receipt of University of Wales studentships. NF acknowledges the receipt of a PPARC studentship. This work was supported by grants GR/H64361 and GR/H86264 from PPARC.

\section{A. Code details}

The value of any function, $A$, at an SPH particle $i$ can be calculated from a sum over neighbouring particles $j$ :

$$
A_{i}=\sum_{j} \frac{m_{j}}{\rho_{j}} A_{j} W\left(\mathbf{r}_{i}-\mathbf{r}_{j}, h\right)
$$

(Monaghan 1992), where $h$ is a scale length for the SPH particles and $W$ a normalised interpolating kernel.

The kernel used in this code is the $M_{4}$ kernel (Monaghan \& Lattanzio 1985) which has been well-tested, and has compact support (i.e. the kernel is finite in extent). The form of the $M_{4}$ kernel is:

$$
M_{4}(u)=\eta_{d} \begin{cases}\frac{2}{3}-u^{2}+\frac{1}{2} u^{3}, & 0 \leq u \leq 1, \\ \frac{1}{6}(2-u)^{3}, & 1 \leq u \leq 2, \\ 0, & 2 \leq u,\end{cases}
$$

where $u=\left|\mathbf{r}_{i}-\mathbf{r}_{j}\right| / h$ and $\eta_{d}$ is a normalisation constant:

$$
\eta_{d}= \begin{cases}1 / h, & d=1, \\ 15 /\left(7 \pi h^{2}\right), & d=2, \\ 3 /\left(2 \pi h^{3}\right), & d=3,\end{cases}
$$

where $d$ is the dimension.

The dimensionless form of the kernel is as above, without the factor of $1 / h^{d}$ in the normalisation constant.

The code uses a spatially-varying smoothing length, $h$. If $h_{i}$ is not equal to $h_{j}$ then particle $i$ may overlap particle $j$ but not vice-versa, so that it is possible for particle $i$ to exert a force on $j$ without $j$ exerting a corresponding reaction on $i$, thereby violating Newton's Third Law. In order to overcome this problem, a mean value of $h$ is used when calculating forces (Benz et al. 1990):

$$
h=\frac{h_{i}+h_{j}}{2} .
$$

The integration scheme used is a second order predictor-corrector with a constant timestep. We use a treecode (Hernquist \& Katz 1989) to find SPH neighbours and to calculate the gravitational forces.

The values of $h$ given to the particles are calculated in two different ways. The first method is to calculate $h$ based upon the density of the particle. If the particles each have mass $m$, and it is desired that each particle should have $N_{\text {kernel }}$ neighbours within its kernel, then the total mass contained within the kernel is $m N_{\text {kernel. If the density at }}$ particle $i$ is $\rho_{i}$ then for the $3 \mathrm{D}$ case:

$$
m N_{\text {kernel }}=\frac{4}{3} \pi\left(2 h_{i}\right)^{3} \rho_{i}
$$

leading to:

$$
h_{i}=\left(\frac{3 m N_{\mathrm{kernel}}}{32 \pi \rho_{i}}\right)^{\frac{1}{3}} .
$$

A similar argument for the $2 \mathrm{D}$ case gives:

$$
h_{i}=\left(\frac{m N_{\mathrm{kernel}}}{4 \pi \sigma_{i}}\right)^{\frac{1}{2}}
$$

where $\sigma_{i}$ is the surface density at particle $i$.

The second method is to set the value of $h$ for each particle so that the number of particles within a distance $h_{i}+h_{j}$ lies within a specified range. The tolerance used is $10 \%$, so that for example if the desired number of neighbours is 50, the values of $h$ are adjusted until each particle has a number of neighbours in the range $50 \pm 5$.

The first method is used in situations in which there are no large changes in density. In this paper it is used in the static tests and in the simulation of Poiseuille flow. The second method is used where it is necessary to model a steep contrast in density, and is used in the shock-tube and in the simulation of the collapse of the isothermal sphere. 


\section{References}

Artymowicz P., Lubow S.H., 1994, ApJ 421, 651

Barnes J., Hut P., 1986, Nat 324, 446

Benz W., Bowers R.L., Cameron A.G.W., Press W.H., 1990, ApJ 348, 647

Brookshaw L., 1985, Proc. Astr. Soc. Austr. 6, 207

Flebbe O., Münzel S., Herold H., et al., 1994, ApJ 431, 754

Frank J., King A.R., Raine D.J., 1992, Accretion Power in Astrophysics. 2nd ed.; Cambridge: Cambridge Univ. Press

Gingold R.A., Monaghan J.J., 1977, MNRAS 181, 375

Hernquist L., Katz N., 1989, ApJS 70, 419

Lucy L.B., 1977, AJ 82, 1013

Lynden-Bell D., Pringle J.E., 1974, MNRAS 168, 603

Maddison S.T., Murray J.R., Monaghan J.J., 1996, Publs. Astron. Soc. Australia 13, 66

Meglicki Z., Wickramasinghe D., Bicknell G.V., 1993, MNRAS 264, 691
Monaghan J.J., 1989, J. Comput. Phys. 82, 1

Monaghan J.J., 1992, ARA\&A 30, 543

Monaghan J.J., Gingold R.A., 1983, J. Comput. Phys. 52, 374

Monaghan J.J., Lattanzio J.C., 1985, A\&A 149, 135

Murray J.R., 1996, MNRAS 279, 402

Pringle J.E., 1981, ARA\&A 19, 137

Sod G.A., 1978, J. Comput. Phys. 27, 1

Takeda H., Miyama S.M., Sekiya M., 1994, Prog. Theor. Phys. 92, 939

Turner J.A., Bhattal A.S., Chapman S.J., et al., 1995, MNRAS 277,705

Whitworth A.P., Bhattal A.S., Chapman S.J., et al., 1995a, MNRAS 277, 727

Whitworth A.P., Bhattal A.S., Turner J.A., Watkins S.J., 1995b, A\&A 301, 929

Wood D., 1981, MNRAS 194, 201 